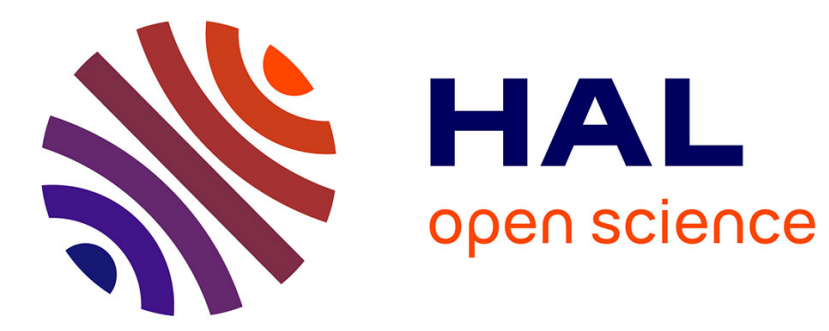

\title{
Aggregation of importance measures for decision making in reliability engineering
}

Michele Compare, Michele Bellora, Enrico Zio

\section{To cite this version:}

Michele Compare, Michele Bellora, Enrico Zio. Aggregation of importance measures for decision making in reliability engineering. Proceedings of the Institution of Mechanical Engineers, Part O: Journal of Risk and Reliability, 2017, 231 (3), pp.242 - 254. 10.1177/1748006X17694495 . hal01652234

\section{HAL Id: hal-01652234 \\ https://hal.science/hal-01652234}

Submitted on 30 Nov 2017

HAL is a multi-disciplinary open access archive for the deposit and dissemination of scientific research documents, whether they are published or not. The documents may come from teaching and research institutions in France or abroad, or from public or private research centers.
L'archive ouverte pluridisciplinaire HAL, est destinée au dépôt et à la diffusion de documents scientifiques de niveau recherche, publiés ou non, émanant des établissements d'enseignement et de recherche français ou étrangers, des laboratoires publics ou privés. 


\title{
Aggregation of Importance Measures for decision making in reliability engineering
}

\author{
Michele Compare ${ }^{1,2}$, Michele Bellora ${ }^{1}$, Enrico Zio ${ }^{1,2,3}$ \\ ${ }^{1}$ Energy Department, Politecnico di Milano, Milan, Italy \\ ${ }^{2}$ Aramis s.r.l., Milan, Italy
}

${ }^{3}$ Chair System Science and the Energy Challenge, Fondation Electricite' de France (EDF), CentraleSupélec, Université Paris-Saclay, Chatenay-Malabry, France

\section{Abstract}

This paper investigates the aggregation of rankings based on component Importance Measures (IMs) to provide the decision maker with a guidance for design or maintenance decisions. In particular, ranking aggregation algorithms of literature are considered, a procedure for ensuring that the aggregated ranking is compliant with the Condorcet criterion of majority principle is presented, and two original ranking aggregation approaches are proposed. Comparisons are made on a case study of an auxiliary feed-water system of a nuclear pressurized water reactor.

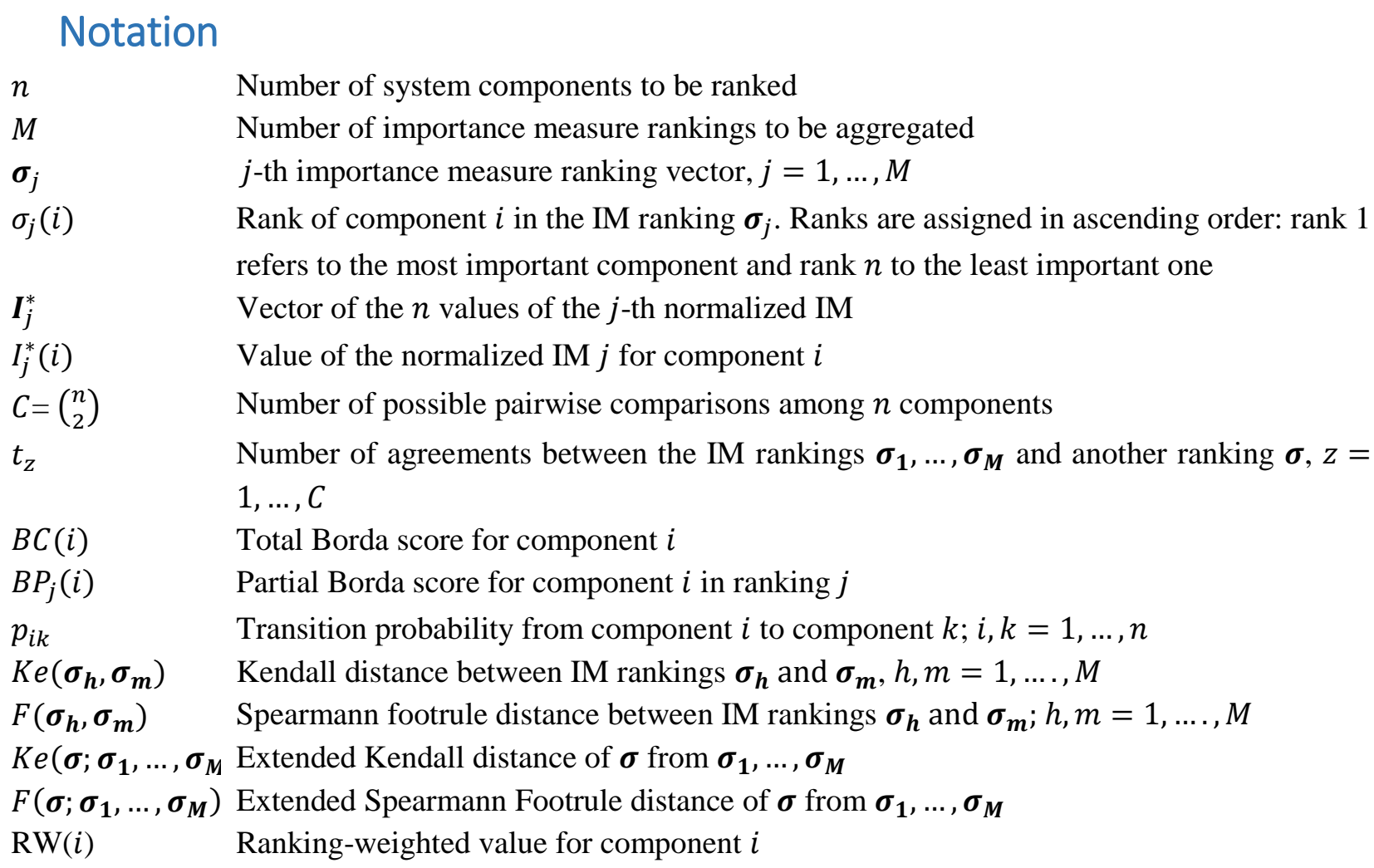




\section{Introduction}

From a broad perspective, the main objectives of system reliability analysis are the estimation of the probability of the system being able to perform its functions for the given mission time [1] and the identification of cost-effective solutions to ensure the expected system reliability with the lowest life cycle cost. Clearly, system reliability mainly depends on the reliability of its constituent parts and the functional, logic and physical relationships among them. Then, a relevant part of reliability analysis consists in describing the relationships (by developing mathematical models) between the stochastic failure behavior of the system and that of its constituent parts. The corresponding reliability models allow also to perform an Importance Measure (IM) analysis [2], which is useful in industrial practice for cost-effective reliability allocation and maintenance strategy definition. Quantitative IMs have been introduced for quantifying the relative importance of the components on the Reliability Availability and Maintainability (RAM) performance of the whole system, depending on the role, function and logic position they have in the system failure structure and/or on their failure probability values.

Various IMs have been proposed in the literature [3]-[12], with different scopes of application. In this respect, reference [12][13] divides IMs into two classes:

1) Design Centered (DC) IMs; these are used to support decisions about system design or redesign (i.e., whether adding or removing components, subsystems, changing operating procedures, etc.), based on the evaluation of the significance of the effect of a component not being in service, due to either a failure or a preventive maintenance action. According to the definition provided in [13], we include in this class the Birnbaum (Bi) and the Risk Reduction Worth (RRW) IMs.

2) Test \& Maintenance Centered (TMC) IMs, which aim at evaluating the impact on the system performance of changes in the test and maintenance strategy on the system components. The Fussel-Vesely (FV), Criticality (Cr) and Risk Achievement Worth (RAW) IMs belong to this class [13].

On the other side, whichever the application, different IMs could lead to different component rankings and, thus, to different design choices (for DC IMs) or test and maintenance policies (for TMC IMs). This poses the challenging decision making problem of how to exploit the possibly conflicting different pieces of information to make the final decision.

To address this issue, which is the motivation of this work, some works of the literature (e.g., [5], [14]) have deeply investigated the relationships between the different IMs. Although these studies give a sound basis to justify the differences among the IM rankings, they do not give a formal approach to decision making.

A different perspective, which is considered in this work, is that of building an absolute ranking of the system components by aggregating the different IM rankings, based on mathematically sound rules. To do this, we propose to frame the IMs as voters that express preferences among the components, which are candidates in the election for being the most important item to focus the attention on for introducing changes in the system design or in the maintenance and test strategy, to improve the system RAM performance. This conceptual framework gives us the possibility of exploiting the mature and wide literature on voting systems. The underlying idea is that an aggregated 
ranking that integrates the pieces of information obtained from all the IMs gives the Decision Maker (DM) the possibility to make more informed decisions.

The first attempts at integrating different opinions about the rank positions of some alternatives date back to the end of the $18^{\text {th }}$ century, at the birth of the modern democracy in France [15], [16], when the philosopher Jean-Jacques Rousseau tried to justify the principle of majority rule underpinning his concept of popular sovereignty [17]. With the same aim, the first theories about rank aggregation were proposed by Condorcet [15], whose key criterion is that a candidate that wins by majority in all pairings against the other candidates is the social best choice. This property has also a statistical justification: suppose that there exists a "true" best candidate, then in the Condorcet scheme he/she is the most likely to be the winner. The drawback of this method lies in that a winner not always exists [18].

Since then, many Ranking Aggregation (RA) methods have been developed, and their mathematical properties formalized. In particular, in the last years RA methods have been proposed to answer a number of modern issues such as the quality assessment of industrial products [19], the feature selection for machine intelligence and data mining [20]-[22], validation measures for clustering algorithms [23], and the integration of ordered list of genes in bioinformatics [24], [25]. Yet, RA algorithms have been extensively studied in the Web context to build meta-search engines or improve search precision through word association [26], [27], and in the context of political sciences to set voting rules in modern democracies [28]. In these fields, a change in the methods to aggregate preferences may have countless implications in our daily life.

In spite of the relevance of these algorithms in the modern society, to the authors' best knowledge they have never been investigated in the context of IMs of engineering equipment, although some of them have been applied to similar engineering issues (e.g., [29], [30]).

On this background, the aim of this paper is to illustrate the application of the theory of RA to the field of reliability analysis, in support to decision making for system redesign or test and maintenance policy making. To do this, we consider four RA techniques of literature together with a procedure to make the aggregated rankings compliant with the Condorcet Criterion (CC) of majority principle. Moreover, we propose two additional RA methods, which are compared with the previous ones by way of a case study concerning the IMs of the components of an Auxiliary Feed-Water System (AFWS) of a nuclear Pressurized Water Reactor (PWR).

Finally, the approach based on IM ranking aggregation is compared with the Multi-Criteria Decision Making (MCDM) approach, where IMs are framed as objective functions to be maximized, and the Pareto sets of components with non-dominated IM values are found [31].

To sum up, the contribution of this work lies in the identification of general rules for taking decisions to improve the system reliability and/or the maintenance strategy. To do this, we consider RA methods typically used in other domains, two original RA methods and the MCDM approach.

The remainder of the paper is as follows. In Section 2, we introduce two measures to evaluate the distance between rankings, which are fundamental to evaluate the performance of the RA methods and are at the basis of some of them. In Section 3, some relevant RA methods of literature are presented together with two original approaches and with a procedure for modifying rankings to satisfy the Condorcet property. The methods described in Section 3 are applied in Section 4 to the 
IMs of the components of the PWR system that serves as case study. A final discussion is presented in Section 5 and conclusions are outlined in Section 6.

\section{Distance between IM rankings}

In this work, we consider different methods to aggregate the rankings obtained from different IMs. To compare their performance, it is fundamental to introduce the concept of distance among rankings. The following two distances are considered in this work, which are derived from [32]:

1. The Kendall Distance (KD), $K e\left(\boldsymbol{\sigma}_{\boldsymbol{h}}, \boldsymbol{\sigma}_{\boldsymbol{m}}\right)$, between IM rankings $\boldsymbol{\sigma}_{\boldsymbol{h}}$ and $\boldsymbol{\sigma}_{\boldsymbol{m}}$ of $n$ components. This distance counts the number of pairwise adjacent transpositions needed to transform one list into the other. Its formal definition is:

$$
K e\left(\boldsymbol{\sigma}_{\boldsymbol{h}}, \boldsymbol{\sigma}_{\boldsymbol{m}}\right)=\llbracket\left\{(i, k) \text { s.t. } \boldsymbol{\sigma}_{\boldsymbol{h}}(i)<\boldsymbol{\sigma}_{\boldsymbol{h}}(k), \boldsymbol{\sigma}_{\boldsymbol{m}}(i)>\boldsymbol{\sigma}_{\boldsymbol{m}}(k)\right\} \rrbracket
$$

where the operator $\llbracket \cdot \rrbracket$ indicates the cardinality of the argument set. The distance $K e$ ranges between zero and $\left(\begin{array}{l}n \\ 2\end{array}\right)$ : the larger its value, the larger the difference between the rankings. For example, consider rankings $\sigma_{1}$ and $\sigma_{2}$ in Table 1: they differ for a swap between the elements A and B only; then, $\operatorname{Ke}\left(\sigma_{1}, \sigma_{2}\right)=1$. Conversely, rankings $\sigma_{1}$ and $\sigma_{3}$ are opposite: then, the maximum number $\left(\begin{array}{l}n \\ 2\end{array}\right)=\frac{5 !}{2 ! \cdot 3 !}=10$ of transpositions is needed to transform a ranking into the other.

\begin{tabular}{|c|l|l|l|l|l|l|l|l|l|l|l|l|l|l|}
\hline & \multicolumn{4}{|l|}{ Alternatives } & \multicolumn{3}{l|}{ Dist. from $\boldsymbol{\sigma}_{\mathbf{1}}$} & \multicolumn{2}{l|}{ Dist. from $\boldsymbol{\sigma}_{\mathbf{2}}$} & \multicolumn{2}{l|}{ Dist. from $\boldsymbol{\sigma}_{\mathbf{3}}$} & \multicolumn{2}{l|}{$\begin{array}{l}\text { Extended } \\
\text { distance }\end{array}$} \\
\hline Rankings & $\mathrm{A}$ & $\mathrm{B}$ & $\mathrm{C}$ & $\mathrm{D}$ & $\mathrm{E}$ & $\mathrm{KD}$ & $\mathrm{SFD}$ & $\mathrm{KD}$ & $\mathrm{SFD}$ & KD & SFD & KD & SFD \\
\hline $\boldsymbol{\sigma}_{\mathbf{1}}$ & $1^{\circ}$ & $2^{\circ}$ & $3^{\circ}$ & $4^{\circ}$ & $5^{\circ}$ & 0 & 0 & 1 & 2 & 10 & 12 & 5.5 & 7 \\
\hline $\boldsymbol{\sigma}_{\mathbf{2}}$ & $2^{\circ}$ & $1^{\circ}$ & $3^{\circ}$ & $4^{\circ}$ & $5^{\circ}$ & 1 & 2 & 0 & 0 & 9 & 12 & 5 & 7 \\
\hline $\boldsymbol{\sigma}_{\mathbf{3}}$ & $5^{\circ}$ & $4^{\circ}$ & $3^{\circ}$ & $2^{\circ}$ & $1^{\circ}$ & 10 & 12 & 9 & 12 & 0 & 0 & 9.5 & 12 \\
\hline
\end{tabular}

Table 1: Example of KD and SFD

2. The Spearman Footrule Distance (SFD), $F\left(\boldsymbol{\sigma}_{\boldsymbol{h}}, \boldsymbol{\sigma}_{\boldsymbol{m}}\right)$, sums the absolute difference between each alternative in rankings $\boldsymbol{\sigma}_{\boldsymbol{h}}$ and $\boldsymbol{\sigma}_{\boldsymbol{m}}$; that is:

$F\left(\sigma_{h}, \sigma_{m}\right)=\sum_{i=1}^{N}\left|\sigma_{h}(i)-\sigma_{m}(i)\right|$

This measure ranges between 0 and $\left\lfloor\frac{n^{2}}{2}\right\rfloor$, where $\lfloor\cdot\rfloor$ indicates the integer part of a real number. From Table 1, we can see that also for SFD the maximum distance value is achieved in case of opposite rankings, although this is not a necessary condition.

Moreover, from Table 1 we can also note that the SFD between two rankings is always larger than or equal to the corresponding KD. This is a general result; namely, the following inequality has been proved [35]:

$$
K e\left(\boldsymbol{\sigma}_{\mathbf{1}}, \boldsymbol{\sigma}_{\mathbf{2}}\right) \leq F\left(\boldsymbol{\sigma}_{\mathbf{1}}, \boldsymbol{\sigma}_{\mathbf{2}}\right) \leq 2 K e\left(\boldsymbol{\sigma}_{\mathbf{1}}, \boldsymbol{\sigma}_{\mathbf{2}}\right)
$$

which states that SFD is an upper bound of KD, smaller than its double.

Finally, both the definitions of KD and SFD can be extended to the distance of a given ranking $\boldsymbol{\sigma}$ from a set of $M$ rankings: 


$$
\begin{gathered}
K e\left(\boldsymbol{\sigma} ; \boldsymbol{\sigma}_{\mathbf{1}}, \ldots, \boldsymbol{\sigma}_{\boldsymbol{M}}\right)=\frac{1}{M} \sum_{j=1}^{M} K e\left(\boldsymbol{\sigma}, \boldsymbol{\sigma}_{\boldsymbol{j}}\right) \\
F\left(\boldsymbol{\sigma} ; \boldsymbol{\sigma}_{\mathbf{1}}, \ldots, \boldsymbol{\sigma}_{\boldsymbol{M}}\right)=\frac{1}{M} \sum_{j=1}^{M} F\left(\boldsymbol{\sigma}, \boldsymbol{\sigma}_{\boldsymbol{j}}\right)
\end{gathered}
$$

In words, the extended distance of $\sigma$ from $\sigma_{1}, \ldots, \sigma_{M}$ is the mean value of its distances from every other IM (Table 1, last column).

As mentioned earlier, these distance measures can be used as performance indicators of the RA algorithms. Intuitively, given a set of IM rankings $\sigma_{1}, \ldots, \sigma_{M}$, which are "voting" the best candidate among all the components, the ranking $\boldsymbol{\sigma}$ that minimizes their mutual distances (e.g., Eq. (4) or Eq. (5)) is the most representative for $\sigma_{1}, \ldots, \sigma_{M}$, according to the considered distance. The smaller the distance, the better the capability of synthesis of the IM rankings. This intuitive reasoning has a statistical formalization [35].

Finally KD and SFD are at the basis of some aggregation methods discussed below.

\section{Aggregation methods}

The aim of this paper is to illustrate some aggregation methods that can be applied to the reliability engineering field in support to decision making based on IMs ranking. For the sake of clarity, all the methods considered are divided into two categories, depending on whether the aggregation works on the IM rank orders or, rather, directly on the IM values:

1) Rank-based methods: each IM gives rise to a ranking of component importance. Then, the rank orders are aggregated forming a unique ranking. In this class, we consider the Borda count method, Markov chain method, Kemeny and Footrule aggregation methods, which are derived from the existing literature, and the original ranking-weighted method.

2) Value-based methods: the component IM values are normalized between 0 and 100 and, then, aggregated according to some rule. The aggregated values are finally ranked in descendent order. In this class, we will present the simple mean method and the value-weighted method.

A detailed description of these methods is given below to highlight their similarity and differences. Then, the methods are compared in Section 4 by means of the example of the AWFS.

Finally notice that similarly to the value-based methods, the MCDM approach is applied on the normalized IM values.

\subsection{Rank-based methods}

\subsubsection{Borda count method}

The Borda count method, which was formalized by Jean-Charles de Borda in 1784 [16], assumes that an alternative is better than another when it gets a higher average position in the different available rankings. In other words, the best alternative is that which on average defeats the largest number of alternatives in the voting profile (i.e., IMs) considered.

Formally, given a set of $M$ IMs for a system made up of $N$ components, the total Borda score $B C(i)$ of the $i$-th component, $i=1, \ldots, n$ is:

$$
B C(i)=\sum_{j=1}^{M} B P_{j}(i)
$$


where the partial score $B P_{j}(i)$ is the number of alternatives in which $i$ is defeated by in IM ranking $\sigma_{j}$.

Finally, the values $B C(i) i=1, \ldots, n$ are sorted to get the final ranking: smaller values correspond to more important components.

For example, consider the rankings in Table 1. Then, $B C(A)=B P_{1}(A)+B P_{2}(A)+B P_{3}(A)=0+$ $1+4=5$.

\subsubsection{Markov chain method}

A new class of aggregation methods based on Markov Chains have been proposed in [32], where the underlying idea is that the alternatives to be ranked correspond to the $n$ states of a Markov Chain whose transition matrix depends on the given rankings. In this setting, the stationary distribution of the chain will provide the aggregated ranking of the components.

According to the findings of [32], we set the transition probabilities of the chain as follows:

$$
p_{i k}=\left\{\begin{array}{ll}
\frac{1}{n} * \frac{\llbracket \text { rankings } \sigma_{j} \text { t.c. } \sigma_{j}(k)<\sigma_{j}(i) \rrbracket}{M} & \text { if } k \neq i \\
1-\sum_{k \neq i} p_{i k} & \text { if } k=i
\end{array} \quad i, k=1, \ldots, n\right.
$$

For example, the transition probability matrix for the rankings in Table 1 reads:

$$
P=\left[\begin{array}{ccccc}
\frac{10}{5 \cdot 3} & \frac{2}{5 \cdot 3} & \frac{1}{5 \cdot 3} & \frac{1}{5 \cdot 3} & \frac{1}{5 \cdot 3} \\
\frac{1}{5 \cdot 3} & \frac{11}{5 \cdot 3} & \frac{1}{5 \cdot 3} & \frac{1}{5 \cdot 3} & \frac{1}{5 \cdot 3} \\
\frac{2}{5 \cdot 3} & \frac{2}{5 \cdot 3} & \frac{9}{5 \cdot 3} & \frac{1}{5 \cdot 3} & \frac{1}{5 \cdot 3} \\
\frac{2}{5 \cdot 3} & \frac{2}{5 \cdot 3} & \frac{2}{5 \cdot 3} & \frac{8}{5 \cdot 3} & \frac{1}{5 \cdot 3} \\
\frac{2}{5 \cdot 3} & \frac{2}{5 \cdot 3} & \frac{2}{5 \cdot 3} & \frac{2}{5 \cdot 3} & \frac{7}{5 \cdot 3}
\end{array}\right]
$$

To wit, the numerator of entry $(1,2)$ of matrix $P$ is 2 because component ' $\mathrm{B}$ ' is more important than 'A' in two rankings (i.e., $\sigma_{2}$ and $\sigma_{3}$ ).

From Eq. (7), it clearly emerges that the Markov chain will visit more frequently the components ranked in the first positions of IMs $\sigma_{1}, \ldots, \sigma_{M}$. For this, the steady state probability values of these top-ranked alternatives will be the largest. The ranking of the steady state probabilities will be the final aggregated ranking, in which the components with the largest probabilities are ranked first. With reference to the example of Table 1 , the steady state vector (i.e., the left eigenvector of $P,[36]$ ) and the corresponding raking vector are $[0.238,0.333,0.179,0.139,0.111]$ and $[2,1,3,4,5]$, respectively.

As emerged in [32], the RA method based on the Markov Chain can be seen as a generalization of the Borda count method. In fact, the probability of staying in state $i, p_{i i}$, is proportional to the number of components that $i$ defeats in the IMs $\sigma_{1}, \ldots, \sigma_{M}$. This concept is similar to that of Borda score $B C(i)$. 
Notice also that there may be recurrent states, which will lead to assign steady state probabilities to the recurrent part of the chain only. When this happens, it suffices to remove from the chain the alternatives already ranked, and repeat the procedure to rank the remaining states [32].

Finally, notice that although the Markov Chain settings is intuitive and has proven to give useful results of ranking aggregation in the Web context [32], it is an heuristics, and a sound mathematical formalization of this algorithm is lacking. The definition of such formalization will be the object of future research work.

\subsubsection{Kemeny and Footrule optimal aggregation techniques}

This method has been proposed by Kemeny in [37] and is based on the following assumptions:

- There is a true, but unknown, ranking $\boldsymbol{\sigma}$ of the importance of the components to improve, for example, the system reliability by its re-design.

- We are given rankings $\boldsymbol{\sigma}_{\mathbf{1}}, \ldots, \boldsymbol{\sigma}_{\boldsymbol{M}}$, which are "noisy" versions of $\boldsymbol{\sigma}$.

- Based on the Condorcet jury theorem [18], the probability $p$ of the IM $\sigma_{j}$ giving the correct, although unknown, order to two alternatives in any pairwise comparison, must be larger than 0.5 . This assumption assures that adding more voters (i.e. IMs) increases the probability that the majority decision is correct. Otherwise, the optimal jury consists of a single voter.

In this setting, the Kemeny optimal RA algorithm searches for the ranking $\overline{\boldsymbol{\sigma}}$ which is the most likely to be $\sigma$.

Formally, let $t_{z}$ be the number of agreements between the voting profile $\boldsymbol{\sigma}_{1}, \ldots, \boldsymbol{\sigma}_{\boldsymbol{M}}$ and a ranking $\boldsymbol{\sigma}$ on the $z$-th pairwise comparison, $z=1, \ldots, C, t_{z} \in\{0,1, \ldots, M\}$. The likelihood function for the ranking $\boldsymbol{\sigma}$ is:

$$
L\left(\boldsymbol{\sigma} ; \boldsymbol{\sigma}_{1}, \ldots, \boldsymbol{\sigma}_{M}\right)=\prod_{z=1}^{C}\left(\begin{array}{l}
M \\
t_{z}
\end{array}\right) p^{t_{z}}(1-p)^{M-t_{z}}
$$

That is, for each pairwise comparison, the number of correct order assignments into the IM rankings $\sigma_{1}, \ldots, \sigma_{M}$, provided that the true order is $\sigma$, has a binomial distribution with parameters $p$ and $M$. Ranking $\overline{\boldsymbol{\sigma}}$ is that which maximizes the total number $\sum_{z=1}^{C} t_{z}$ of agreements with the available rankings. In other words, we are searching for ranking $\overline{\boldsymbol{\sigma}}$, which maximizes the probability of having sampled $\sigma_{1}, \ldots, \sigma_{M}$, provided that the probability of each IM $\sigma_{j}, j=1, \ldots M$, assigning the correct order to two alternatives in any pairwise comparison, is $p>0.5$ [32].

Ranking $\overline{\boldsymbol{\sigma}}$ is also called Kemeny optimal aggregation, because it has been proved that it minimizes the distance $K e\left(\cdot ; \boldsymbol{\sigma}_{\mathbf{1}}, \ldots, \boldsymbol{\sigma}_{\boldsymbol{M}}\right)$ [39]. However, in [32] it has also been proved that the computation of the Kemeny optimal aggregation is NP-hard and, thus, there is no algorithm that allows efficiently working with the Kemeny distance. For this reason, the algorithms to find $\bar{\sigma}$ are not investigated here; rather, to overcome this limitation we consider the SFD. That is, we find the footrule optimal aggregation ranking $\widetilde{\boldsymbol{\sigma}}$ minimizing $F\left(\cdot ; \boldsymbol{\sigma}_{\mathbf{1}}, \ldots, \boldsymbol{\sigma}_{\boldsymbol{M}}\right)$, which is an approximation of the actual optimum Kemeny ranking $\overline{\boldsymbol{\sigma}}$. In fact, the following inequality has been proved in [35], which directly stems from Eq. (3):

$$
K e\left(\overline{\boldsymbol{\sigma}} ; \boldsymbol{\sigma}_{1}, \ldots, \boldsymbol{\sigma}_{\boldsymbol{M}}\right) \leq K e\left(\widetilde{\boldsymbol{\sigma}} ; \boldsymbol{\sigma}_{\mathbf{1}}, \ldots, \boldsymbol{\sigma}_{\boldsymbol{M}}\right) \leq 2 K e\left(\overline{\boldsymbol{\sigma}} ; \boldsymbol{\sigma}_{\mathbf{1}}, \ldots, \boldsymbol{\sigma}_{\boldsymbol{M}}\right)
$$


In words, the Kemeny distance of $\widetilde{\boldsymbol{\sigma}}$ from the given rankings is an upper bound of the same Kemeny distance of $\bar{\sigma}$ from the same rankings $\sigma_{1}, \ldots, \sigma_{M}$.

The algorithm to find $\widetilde{\boldsymbol{\sigma}}$, which can be computed in polynomial time [32], exploits some notions of graph theory. In the following, we give a pseudo-code of this algorithm, the task of a detailed theoretical study for its justification being left to the interested researchers.

i. Construct a weighted complete bipartite graph $(\Gamma, \Omega, \mathrm{W})$

a. $\quad \Gamma$ is the set of $n$ nodes, which are the components to be ranked.

b. $\Omega$ is the second set of $n$ nodes, which are the available positions $\Omega=\{1, \ldots, n\}$.

c. For every $\gamma \in \Gamma$, and $\omega \in \Omega$ compute weights:

$$
W(\gamma, \omega)=\sum_{j=1}^{M}\left|\sigma_{j}(\gamma)-\omega\right|
$$

ii. Solve the minimum cost perfect matching problem. Namely, a matching $\Psi$ is a collection of edges such that every vertex of $\Gamma \cup \Omega$ is incident to at most one edge of $\Psi$. A matching is perfect if its cardinality is equal to $\llbracket \Gamma \rrbracket=\llbracket \Omega \rrbracket$. The minimum cost perfect matching problem consists in finding the matching which minimizes the cost $\sum_{\psi \in \Psi} W(\gamma, \omega)$, provided that $\gamma, \omega$ are vertices incident to $\psi \in \Psi$. In this work, we have adopted the Hungarian algorithm [33] to address this minimization problem (see [34] for further details).

For example, for the three rankings in Table 1, the weight matrix reads:

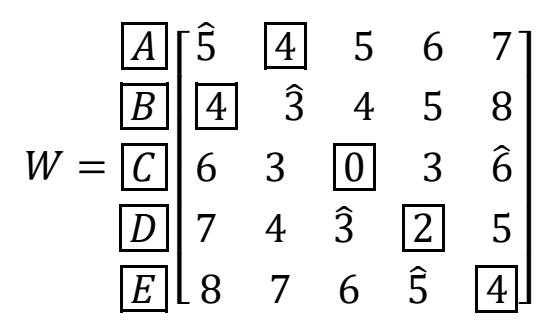

To wit, the $(1,1)$ entry is given by $W(1,1)=|1-1|+|2-1|+|5-1|=5$. The Hungarian algorithm finds the assignment of components to ranking positions, which minimizes the sum of the weights. In this case, it finds the rounded entries of matrix $W$, with total weight equal to 14. A different perfect matching would have been that indicated by hats. Although this matching is perfect, it is not minimal as it sums to 22 . Finally, the ranking positions $\Omega$ are indicated by the column numbers of $W$; then, for every column, the number the row of the corresponding entry selected by the Hungarian algorithm gives the component to be assigned to the position. In the reference example, the row of the entry selected by the algorithm in column 1 of $W$ is equal to 2 . Then, component ' $\mathrm{B}$ ' is assigned position 1 .

\subsubsection{Ranking-weighted method}

In Section 3.1.3, a statistical interpretation has been given to RA, which is based on the idea that all the IM rankings are noisy versions of the "true" unknown order. From this, it follows that a ranking far from the other can be framed as an 'outlier', which is not representative of the population of the noisy rankings and, thus, can introduce a bias in the final ranking. To consider this aspect, we can weigh the rankings to be aggregated depending on their mutual distances.

In details, we propose to compute for each component the weighted sum of its position in the available rankings: 


$$
R W(i)=\sum_{j=1}^{M} w_{j} \sigma_{j}(i)
$$

where weights $w_{j}, j=1, \ldots, M$ are defined as

$$
w_{j}=\frac{1}{N C} \cdot \frac{1}{F\left(\sigma_{j} ; \sigma_{1}, \ldots, \sigma_{j-1}, \sigma_{j+1}, \ldots \sigma_{M}\right)}
$$

and $N C$ is a normalization constant:

$$
N C=\sum_{j=1}^{M} \frac{1}{F\left(\sigma_{j} ; \sigma_{1}, \ldots, \sigma_{j-1}, \sigma_{j+1}, \ldots \sigma_{M}\right)}
$$

This way, the weight assigned to each IM is inversely proportional to its generalized SFD distance from the other IMs, so that a ranking far from the others gets less importance. The components are finally ranked according to their $R W(i)$ value, $i=1, \ldots, n$ : the larger the $\mathrm{RW}$, the smaller the importance of the component.

Notice that the method proposed in this Section, which computes a weighted mean of the rank orders in $\sigma_{1}, \ldots, \sigma_{M}$, can be regarded as a generalization of the Borda count method presented in Section 3.1.1, which is a mean with equal weights.

\subsection{Value-based methods}

The IM ranking methods discussed above are based on the rank of the components, only. That is, to get the final aggregated ranking one focuses on if a component has an IM value larger than the others in the IM rankings available. This way, the actual values of the IMs are disregarded and, thus, the information is lost about how much distant two IM values are. For this reason, we propose intuitive methods based on the values of the IMs of the components, rather than on their ranking positions. These methods are also computationally inexpensive.

\subsubsection{Simple mean method}

The first value-based method is very simple and intuitive: given $m$ vectors of normalized IMs of $n$ components, $\boldsymbol{I}_{\mathbf{1}}^{*}, \ldots, \boldsymbol{I}_{\boldsymbol{M}}^{*}$, we compute the mean of the IM values for each component $i$ :

$$
I^{\text {mean }}(i)=\frac{1}{M} \sum_{j=1}^{M} I_{j}^{*}(i)
$$

Then, the $n$ values $I^{\text {mean }}(i)$ are ranked in descendent order: the larger the value, the smaller the rank. Due to its simplicity, this algorithm is not considered as an original contribution of this work: although we have not found any work of the literature adopting this model, we expect that it has already been used.

\subsubsection{Value-weighted method}

This method is a mixture between the methods presented in Sections 3.1.4 and 3.2.1. Namely, we compute the weighed mean of the values of the normalized IMs:

$$
I^{V}(i)=\sum_{j=1}^{M} w_{j} I_{j}^{*}(i)
$$

with the weight values calculated as in Eq. (11). A drawback in using this method could arise from the fact that IMs like RAW and RRW can assume very large values (even infinite). This entails that all the other components get values next to zero when normalized, even if their original measures 
were different. That is, RAW and RRW may bias the information on the mutual distance of the IMs values. This cannot happen with Birnbaum or Fussel-Vesely measures, since they are limited.

\subsection{Local Kemenization}

Whichever the RA method is, it may be asked to be compliant with a commonly accepted principle in voting: if an alternative is preferred against all the others in a pairwise competition by the majority of the voters, this alternative must be ranked first. This property is called Condorcet criterion (CC), and the alternative chosen is the Condorcet winner. An alternative that respects this property does not necessarily exist, because the voting profile could lead to a loop in the majority relation (e.g., alternative 'A' defeats ' $B$ ' that defeats ' $C$ ' that defeats 'A'). For this reason, Truchon ([39]) proposed the Extended Condorcet Criterion (ECC) as a generalization of the $\mathrm{CC}$ : if there is a partition $(X, \bar{X})$ of the alternatives such that $\forall A \in X, \forall B \in \bar{X}, A$ is preferred to $B$ by the majority of voters, then the elements in $X$ must be ranked smaller (i.e., more important) than those in $\bar{X}$. Again, this partition does not always exist; however, if it does, then it allows identifying a subset of elements that occupy the highest positions in the final ranking, thus giving a partial order to the alternatives.

In this context, the Local Kemenization (LK) [32] is a procedure that modifies an aggregated ranking $\boldsymbol{\sigma}$ so that it accomplishes the ECC with respect to the starting IM rankings $\boldsymbol{\sigma}_{\mathbf{1}}, \ldots, \boldsymbol{\sigma}_{\boldsymbol{m}}$.

Moreover, the modified ranking $\boldsymbol{\sigma}^{\prime}$ is maximally consistent with $\boldsymbol{\sigma}$ and $\boldsymbol{\sigma}_{\mathbf{1}}, \ldots, \boldsymbol{\sigma}_{\boldsymbol{m}}$. That is, if $\boldsymbol{\sigma}^{\prime}(i)<$ $\boldsymbol{\sigma}^{\prime}(j)$, then one of the following propositions hold:

1) $\boldsymbol{\sigma}(i)<\boldsymbol{\sigma}(j)$,

2) A majority of $\sigma_{1}, \ldots, \sigma_{m}$ prefers $i$ to $j$.

In other words, the order of two elements differs between $\boldsymbol{\sigma}$ and $\boldsymbol{\sigma}^{\prime}$ only if the majority of the IM rankings support the change.

The LK procedure proceeds inductively: assume that we have already built the new modified ranking $\boldsymbol{\sigma}^{\prime}$ for the first $i-1$ components of the ranking $\boldsymbol{\sigma}$. Then, the $i$-th component is appended at the end of the actual partial ranking $\boldsymbol{\sigma}^{\prime}$, and it is moved up in the ranking until we find a component that has a majority of votes against it by the original IM rankings $\sigma_{1}, \ldots, \sigma_{m}$.

As the name implies, the ranking $\boldsymbol{\sigma}^{\prime}$ derived from this method is locally Kemeny optimal: there is no ranking $\boldsymbol{\sigma}^{\prime \prime}$ such that $K e\left(\boldsymbol{\sigma}^{\prime}, \boldsymbol{\sigma}^{\prime \prime}\right)=1$, for which $K e\left(\boldsymbol{\sigma}^{\prime \prime} ; \boldsymbol{\sigma}_{1}, \ldots, \boldsymbol{\sigma}_{\boldsymbol{m}}\right)<K e\left(\boldsymbol{\sigma}^{\prime} ; \boldsymbol{\sigma}_{1}, \ldots, \boldsymbol{\sigma}_{m}\right)$. Obviously, the Kemeny optimal aggregation $\overline{\boldsymbol{\sigma}}$ found in Section 3.1.3 is also locally optimal, the converse being not always true.

\section{Case study}

In this Section, we apply the 6 methods described in the previous section also with LK to the IMs of the components of a simplified scheme of an AFWS in a PWR. The example is taken from [13], together with its Reliability Block Diagram (RBD) (Figure 1). The underlying assumption is that all components are in standby mode and periodically tested. This allows predicting the average unavailability of each component as [13]:

$U=\frac{1}{2} \lambda T_{0}+F_{r} \frac{T_{r}}{T}+\frac{T_{t}}{T}$ 
where, $\lambda$ is the component failure rate $\left(\mathrm{h}^{-1}\right)$, $\mathrm{T}$ is the test interval $(\mathrm{h}), T_{R}$ is the average repair duration (h), $T_{t}$ is the average test duration (h), $F_{r}$ is the frequency of repair/test interval and $T_{0}=T-T_{R}-T_{t}$ is the operating time $(\mathrm{h})$. The values of these parameters are reported in Table 2.

Figure 2 shows the normalized values of the Bi, RRW, FV, Cr and RAW IMs. In particular, the value $I_{j}(i)$ of the $j$-th IM of the $i$-th component, $i=1 \ldots, n$, and $j=1, \ldots, M$ is normalized between 0 and 100 as follows:

$$
I_{j}^{*}(i)=\frac{I_{j}(i)-\min _{i}\left(I_{j}(i)\right)}{\max _{i}\left(I_{j}(i)\right)-\min _{i}\left(I_{j}(i)\right)} * 100
$$

The corresponding IM rankings are reported in Table 3.

From Figure 2, we can note that some of the IMs considered give similar results in evaluating the importance of the components. For example, Bi and RAW IMs give the components the same rank positions: component ' $\mathrm{N}$ ' is much more relevant than any other, whereas ' $\mathrm{L}$ ' stands out from the remaining ones (mainly due to the larger unavailability of ' $M$ '). Also RRW and $\mathrm{Cr}$ give the components the same ranking orders (Table 3), even if the IM values are slightly different (i.e., the values of Cr for components 'I', 'J', 'K' and 'L' are larger than the corresponding values of RRW). The FV measure gives results similar to those of RRW and Cr, except for component ' $N$ ', which is given the third position.

To sum up the results in Table 3, the five IMs considered assign the same ranking positions to the last six components, only, whereas the rank orders differ for the most important ones. This entails that it is not possible to make a simple decision on system design and maintenance, as if the rankings were the same. This consideration also holds if we divide the IMs into the two categories proposed in Section 3: rankings in the first two rows (DC IMs) are different from each other as well as those in the last three rows (TMC IMs). Thus, to make a decision we consider the aggregation of the rankings.

Finally, notice that as it clearly emerges from the system RBD (Figure 1) and component parameter values (Table 2), components 'I' and ' $J$ ' can be inter-changed without altering the system reliability behavior. Then, their ranks coincide in every IM. However, without loss of generality, in each ranking we consider component ' $\mathrm{J}$ ' less important than component 'I'.

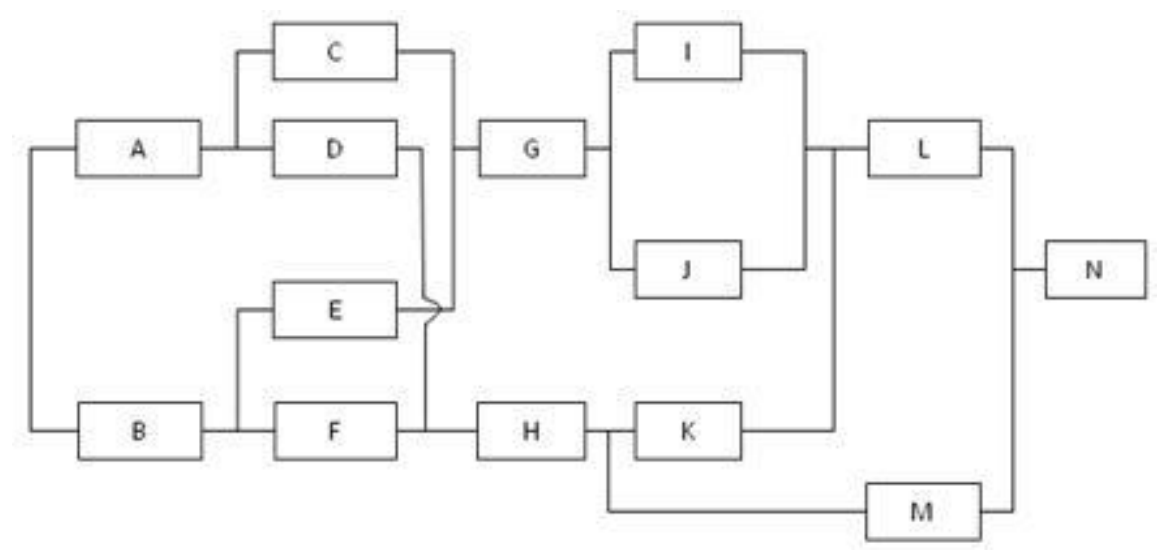




\begin{tabular}{|l|l|l|l|l|l|l|l|l|l|l|l|l|l|l|}
\hline Component & A & B & C & D & E & F & G & H & I & J & K & L & M & N \\
\hline \multicolumn{1}{|l|}{$\lambda$} & $1.0 \mathrm{E}-7$ & $1.0 \mathrm{E}-7$ & $1.0 \mathrm{E}-6$ & $1.0 \mathrm{E}-6$ & $1.0 \mathrm{E}-6$ & $1.0 \mathrm{E}-6$ & $1.0 \mathrm{E}-7$ & $1.0 \mathrm{E}-7$ & $1.0 \mathrm{E}-4$ & $1.0 \mathrm{E}-4$ & $1.0 \mathrm{E}-5$ & $5.0 \mathrm{E}-7$ & $3.0 \mathrm{E}-4$ & $1.0 \mathrm{E}-7$ \\
\hline $\mathrm{F}_{\mathrm{r}}$ & $9.2 \mathrm{E}-3$ & $9.2 \mathrm{E}-3$ & $2.5 \mathrm{E}-2$ & $2.5 \mathrm{E}-2$ & $2.5 \mathrm{E}-2$ & $2.5 \mathrm{E}-2$ & $7.7 \mathrm{E}-4$ & $1.8 \mathrm{E}-4$ & $6.8 \mathrm{E}-1$ & $6.8 \mathrm{E}-1$ & $5.5 \mathrm{E}-1$ & $4.3 \mathrm{E}-3$ & $1.5 \mathrm{E}-1$ & $5.8 \mathrm{E}-4$ \\
\hline $\mathrm{T}_{\mathrm{t}}$ & 0 & 0 & 0 & 0 & 0 & 0 & 0 & 0 & 2 & 2 & 2 & 0 & 0 & 0 \\
\hline $\mathrm{T}_{\mathrm{r}}$ & 5 & 5 & 10 & 10 & 10 & 10 & 15 & 24 & 36 & 36 & 24 & 10 & 10 & 5 \\
\hline $\mathrm{T}$ & 720 & 720 & 720 & 720 & 720 & 720 & 720 & 720 & 720 & 720 & 720 & 720 & 720 & 720 \\
\hline
\end{tabular}

Table 2 AFW component parameters
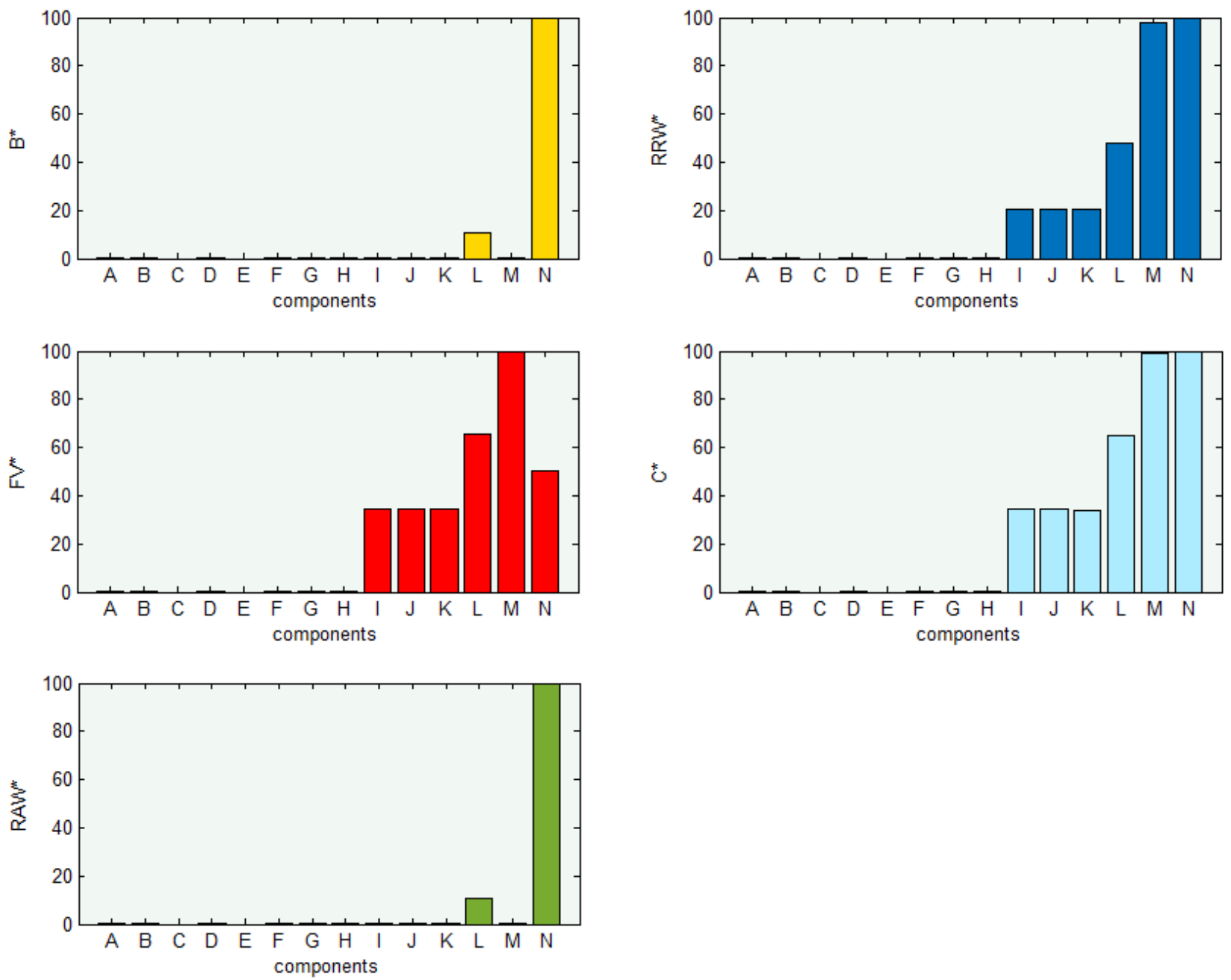

Figure 2 Normalized IMs of the components of the system

\begin{tabular}{|c|cccccccccccccc|}
\hline & $1^{\circ}$ & $2^{\circ}$ & $3^{\circ}$ & $4^{\circ}$ & $5^{\circ}$ & $6^{\circ}$ & $7^{\circ}$ & $8^{\circ}$ & $9^{\circ}$ & $10^{\circ}$ & $11^{\circ}$ & $12^{\circ}$ & $13^{\circ}$ & $14^{\circ}$ \\
\hline B & N & L & H & G & K & M & I & J & A & B & D & F & C & E \\
RRW & N & M & L & I & J & K & H & G & A & B & D & F & C & E \\
\hline
\end{tabular}




\begin{tabular}{|c|cccccccccccccc|}
\hline FV & M & L & N & I & J & K & H & G & A & B & D & F & C & E \\
C & N & M & L & I & J & K & H & G & A & B & D & F & C & E \\
RAW & N & L & H & G & K & M & I & J & A & B & D & F & C & E \\
\hline
\end{tabular}

Table 3 IM rankings: the design-centered IMs are dark grey, whereas the test \& maintenance centered IMs are light grey

\subsection{Results}

In this Section, we apply the six methods presented in Section 3 to aggregate the five IM rankings in the following three settings:

1) Considering all the five IMs

2) Considering only the DC IMs

3) Considering only the TMC IMs

The obtained aggregated rankings are also "locally Kemenized" with the procedure introduced in Section 3.3, and the twelve resulting rankings are compared.

\subsubsection{Application to all the considered IMs}

In this Section, we apply the six aggregation methods presented in Section 3 to the five IM rankings reported in Table 3. Notice that making the final decision about how to improve the system based on the aggregation of DC and TMC IMs must not be surprising: as mentioned above, relationships exist among the IMs of the two groups, which have been deeply investigated in sound works of literature (e.g., [5], [14]). For example (e.g., see [2]), Criticality IM (i.e., a TMC IM) can be regarded as a function of the Birnbaum IM (i.e., a DC IM) as well as FV (i.e., TMC) is linked to RRW (i.e., DC). On this basis, some generalized IMs have also been proposed, which encode both DC and TMC IMs (e.g., [5], [14]).

The aggregated rankings are shown in Table 4 (rows 1-6), which also reports the Kendall generalized distance (Eq. (4)) of every aggregated ranking from the five IMs (last column). As mentioned before, this distance can be regarded as a performance indicator of the aggregation method: the smaller its value, the better is the synthesis of the input rankings.

Table 4 also reports the ranking obtained by applying LK to the six aggregated ranking (last row). The result of the procedure is shown only once because LK yields the same result when it is applied to the six aggregated rankings. Notice also that this ranking has the smallest Kemeny distance together with the two value-based methods and footrule aggregation method; then, it is the most proximal to the available voting profiles. Also, according to the statistical interpretation of the Kemeny distance introduced in Section 2, this ranking is the most likely to be the "true" ranking.

Notice that all the aggregate methods agree that there are three groups of components of different importance: i) components 'L', ' $M$ ' and ' $N$ ' are the most impactful (dark grey area in Table 4); ii) components ' $\mathrm{G}$ ', ' $\mathrm{H}$ ', 'I', 'J', and ' $\mathrm{K}$ ' have a medium level of importance (light grey area in Table 4 ); iii) the remaining six components, 'A', 'B', 'C', 'D', 'E' and ' $F$ ', have the smallest importance (remaining part of Table 4). This information may be useful for decision making. 


\begin{tabular}{l|ccc|ccccc|cccccc|c|}
\cline { 2 - 9 } Borda count & N & L & M & I & H & K & J & G & A & B & D & F & C & E & 6 \\
Markov chain & N & L & M & H & K & G & I & J & A & B & D & F & C & E & 6,8 \\
Footrule aggregation & N & M & L & I & J & K & H & G & A & B & D & F & C & E & 5,2 \\
Ranking-weighted & N & L & M & I & K & H & J & G & A & B & D & F & C & E & 5,8 \\
Simple mean & N & M & L & I & J & K & H & G & A & B & D & F & C & E & 5,2 \\
Values-weighted & N & M & L & I & J & K & H & G & A & B & D & F & C & E & 5,2 \\
Local kemenization & N & M & L & I & J & K & H & G & A & B & D & F & C & E & 5,2 \\
\cline { 2 - 12 }
\end{tabular}

Table 4 Aggregation methods considering all the five IMs

The application of MCDM approach yields the Pareto sets indicated in Table 5. From this Table, it emerges that the Pareto set contains the three components identified as most important by all the aggregation methods (i.e., 'L', 'M', 'N'). The second set of non-dominated solutions contains the components of the medium level of importance, except for ' $\mathrm{J}$ ', which belongs to the third nondominated set. This means that the MCDM approach distinguishes between the importance of this component and those of the components in the previous set, differently from the aggregated rankings. Finally, the last three sets contain the remaining components, ordered coherently with the IM aggregated rankings

\begin{tabular}{|l|c|c|c|c|c|c|}
\hline & 1 & 2 & 3 & 4 & 5 & 6 \\
\hline MCDM & L, M, N & G, H, I, K & J & A, B & D, F & C, E \\
\hline
\end{tabular}

\subsubsection{Application to DC IMs}

Design-centered IMs include the Birnbaum and RRW IMs, which are aggregated in Table 6 by the six RA algorithms considered. When only two IMs are considered, LK does not modify the aggregated rankings, because the Condorcet criterion is always respected. For this reason, the results of the application of LK are not shown in Table 6. In details, consider two generic alternatives ' $A$ ' and ' $\mathrm{B}$ '; then, pairwise comparison must yield one out of the following three outcomes:

1) Both the measures prefer A to B.

2) Both the measures prefer $B$ to $A$.

3) One measure prefers $\mathrm{A}$ to $\mathrm{B}$ while the other prefers $\mathrm{B}$ to $\mathrm{A}$.

In the first two cases, all the aggregation methods obviously rank the preferred alternative higher than the other one (according to the Condorcet criterion), whereas in the third case a Condorcet winner does not exist, although it can be demonstrated that the ECC is always respected.

We can also notice that all the aggregation methods confirm the results of the possibility of partitioning the components according to their importance in three groups, as for the case when all the IM rankings are considered. Moreover, the value-based methods and the Markov Chain method give exactly the same rankings obtained when considering the five IMs.

Furthermore, notice that the generalized Kendall distance of the aggregated rankings is always equal to six. This result is not surprising. In fact, the Kendall distance between the two rankings of the IMs 
is equal to 12. Now, any aggregation method must find a ranking "between" the two input rankings, and a ranking with Kendal distance from the input rankings equal to six can be considered as a 'mean' ranking.

\begin{tabular}{|c|c|c|c|c|c|c|c|c|c|c|c|c|c|c|c|}
\hline DESIGN-CENTERED IMs & $1^{\text {st }}$ & $2^{\text {nd }}$ & $3^{\text {rd }}$ & $4^{\text {th }}$ & $5^{\text {th }}$ & $6^{\text {th }}$ & $7^{\text {th }}$ & $8^{\text {th }}$ & $9^{\text {th }}$ & $10^{\text {th }}$ & $11^{\text {th }}$ & $12^{\text {th }}$ & $13^{\text {th }}$ & $14^{\text {th }}$ & $\mathrm{KD}$ \\
\hline $\mathrm{Bi}$ & $\mathrm{N}$ & $\mathrm{L}$ & $\mathrm{H}$ & G & $\mathrm{K}$ & $\bar{M}$ & $\bar{I}$ & $\overline{\mathrm{J}}$ & A & B & $\mathrm{D}$ & $\overline{\mathrm{F}}$ & $\mathrm{C}$ & $E$ & \\
\hline RRW & $\mathrm{N}$ & M & $\mathrm{L}$ & $\mathrm{I}$ & $\mathrm{J}$ & $\mathrm{K}$ & $\mathrm{H}$ & G & A & B & $\mathrm{D}$ & $\mathrm{F}$ & $\mathrm{C}$ & E & \\
\hline Borda count & $\mathrm{N}$ & $\mathrm{L}$ & M & $\mathrm{H}$ & I & $\mathrm{K}$ & G & $\mathbf{J}$ & A & B & $\mathrm{D}$ & $\mathrm{F}$ & $\mathrm{C}$ & $\mathrm{E}$ & 6 \\
\hline Markov chain & $\mathrm{N}$ & $\mathrm{L}$ & M & $\mathrm{H}$ & $\mathrm{K}$ & G & I & $\mathrm{J}$ & A & B & $\mathrm{D}$ & $\mathrm{F}$ & $\mathrm{C}$ & $\mathrm{E}$ & 6 \\
\hline Footrule aggregation & $\mathrm{N}$ & M & $\mathrm{L}$ & I & K & $\mathrm{J}$ & $\mathrm{H}$ & $\mathrm{G}$ & A & B & $\mathrm{D}$ & $\mathrm{F}$ & $\mathrm{C}$ & $\mathrm{E}$ & 6 \\
\hline Ranking-weighted & $\mathrm{N}$ & $\mathrm{L}$ & M & $\mathrm{H}$ & I & $\mathrm{K}$ & G & $\mathrm{J}$ & $\mathrm{A}$ & B & $\mathrm{D}$ & $\mathrm{F}$ & $\mathrm{C}$ & $\mathrm{E}$ & 6 \\
\hline Simple mean & $\mathrm{N}$ & M & $\mathrm{L}$ & I & $\mathrm{J}$ & $\mathrm{K}$ & $\mathrm{H}$ & $\mathrm{G}$ & A & B & $\mathrm{D}$ & $\mathrm{F}$ & $\mathrm{C}$ & $\mathrm{E}$ & 6 \\
\hline Values-weighted & $\mathrm{N}$ & M & $\mathrm{L}$ & I & $\mathrm{J}$ & K & $\mathrm{H}$ & $\mathrm{G}$ & A & B & D & $\mathrm{F}$ & $\mathrm{C}$ & E & 6 \\
\hline
\end{tabular}

Table 6: Design-centered IMs and relative aggregation methods

Finally, the results of the MCDM approach for this 2-objectives problem are summarized in Table 7. The Pareto set contains component 'N', only; this result is coherent with the Ras in Table 6, where ' $\mathrm{N}$ ' always occupies the first ranking position. The rest of the components are arranged as in the case of all the considered IMs (Table 5).

\begin{tabular}{|l|c|c|c|c|c|c|c|}
\hline & 1 & 2 & 3 & 4 & 5 & 6 & 7 \\
\hline MCDM & $\mathrm{N}$ & $\mathrm{M}, \mathrm{L}$ & $\mathrm{G}, \mathrm{H}, \mathrm{I}, \mathrm{K}$ & $\mathrm{J}$ & $\mathrm{A}, \mathrm{B}$ & $\mathrm{D}, \mathrm{F}$ & $\mathrm{C}, \mathrm{E}$ \\
\hline
\end{tabular}

Table 7: Design-centered IMs and relative aggregation methods

\subsubsection{Applicaton to TMC IMs}

TMC IMs include Fussell-Vesely, RAW and Criticality IMs, whose aggregated rankings are shown in Table 8. These reflect the general results in Table 4: again the footrule aggregation method and the value-based methods agree with the local Kemenization (which does not vary among the six methods), and they are the methods with the minimum Kendall distance from the voting profile.

\begin{tabular}{lllllllllllllll|l|l|} 
TMC IMs & $1^{\text {st }}$ & $2^{\text {nd }}$ & $3^{\text {rd }}$ & $4^{\text {th }}$ & $5^{\text {th }}$ & $6^{\text {th }}$ & $7^{\text {th }}$ & $8^{\text {th }}$ & $9^{\text {th }}$ & $10^{\text {th }}$ & $11^{\text {th }}$ & $12^{\text {th }}$ & $13^{\text {th }}$ & $14^{\text {th }}$ & KD \\
\cline { 2 - 4 } & M & L & N & I & J & K & H & G & A & B & D & F & C & E & \\
FAW & N & L & H & G & K & M & I & J & A & B & D & F & C & E & \\
Rr & N & M & L & I & J & K & H & G & A & B & D & F & C & E & \\
Borda & N & L & M & I & H & K & J & G & A & B & D & F & C & E & 6 \\
Markov chain & N & L & M & K & H & G & I & J & A & B & D & F & C & E & 7 \\
Footrule aggregation & N & M & L & I & J & K & H & G & A & B & D & F & C & E & 4.67 \\
Ranking- weighted & N & L & M & I & J & K & H & G & A & B & D & F & C & E & 5 \\
Simple mean & N & M & L & I & J & K & H & G & A & B & D & F & C & E & 4.67 \\
Values-weighted & N & M & L & I & J & K & H & G & A & B & D & F & C & E & 4.67 \\
Local kemenization & N & M & L & I & J & K & H & G & A & B & D & F & C & E & 4.67 \\
\hline
\end{tabular}


The MCDC approach yields the Pareto sets identified in Table 5. In this respect, it is worth noticing that the aggregation methods with smaller $\mathrm{KD}$ consider component ' $\mathrm{J}$ ' more important than ' $\mathrm{K}$ ' and ' $\mathrm{H}$ ', whereas the opposite conclusion can be derived from Table 5.

\section{Discussion}

Based on the results above, it could seem that after considering different aggregation methods we are back to square one: the aggregated rankings give different results and the decision maker still cannot take a univocal decision. To overcome this issue, in this Section we derive and justify some general rules to provide reliability engineers with a systematic framework for ranking components based on their importance values.

To do this, we first find the 'true' component importance ranking by reducing the failure rate of each component by $\Delta=5-10-20-30 \%$ and, then, we sort the system components according to the extent to which this reduction increases the system availability. This approach gives the true ranking for both DC and TMC IMs: although for TMC IMs we should evaluate the effect on the overall system performance of a change in the parameters relevant to test and maintenance strategy (i.e., $T$, $F_{r}$, etc.), when we do this we always get a reduction in component unavailability, which is the same effect of the reduction in the component failure rates. For this reason, we can assume that the large change (up to $30 \%$ ) in the failure rate encodes all possible changes in the test and maintenance parameters and, thus, the final ranking can be assumed the true ranking also for TMC IMs.

The true ranking is reported in Table 9, which is the same for all considered values of $\Delta$. This ranking corresponds to that of the value-based methods, the Footrule aggregation (except for the DC IMs) and Kemenization (when it makes sense).

With respect to the MCDM approach, the relative importance of component ' $\mathrm{J}$ ' provided in case of both all considered IMs and TMC IMs differs from that in Table 9. This suggests that MCDM can give incorrect results. Besides that, a further pitfall of MCDM for IM-based decision making is that it does not differentiate among the components of the same set: should this contain a large number of components, we would always need an additional technique to support the final decision about the components on which it is most convenient to allocate the budget to improve their reliability or maintenance. For this, the RA approach can to be preferred to MCDM.

\begin{tabular}{|l|l|l|l|l|l|l|l|l|l|l|l|l|l|l|}
\hline RESULTS & $1^{\text {st }}$ & $2^{\text {nd }}$ & $3^{\text {rd }}$ & $4^{\text {th }}$ & $5^{\text {th }}$ & $6^{\text {th }}$ & $7^{\text {th }}$ & $8^{\text {th }}$ & $9^{\text {th }}$ & $10^{\text {th }}$ & $11^{\text {th }}$ & $12^{\text {th }}$ & $13^{\text {th }}$ & $14^{\text {th }}$ \\
\hline Impact ranking & $\mathrm{N}$ & $\mathrm{M}$ & $\mathrm{L}$ & $\mathrm{I}$ & $\mathrm{J}$ & $\mathrm{K}$ & $\mathrm{H}$ & $\mathrm{G}$ & $\mathrm{A}$ & $\mathrm{B}$ & $\mathrm{D}$ & $\mathrm{F}$ & $\mathrm{C}$ & $\mathrm{E}$ \\
\hline
\end{tabular}

Table 9 Impact on system reliability of an increase of up to $30 \%$ of component failure rate

In conclusion, the following practical decision rule is derived from the considerations above:

- In case there are two IMs only, then consider the value-based methods, which are the novelty of this work. They seem to give the most reliable solutions, because in case of contradiction of the two input rankings about two alternatives, they prefer the one which mostly defeats the other. 
- In case there are multiple IMs, choose any aggregation ranking, preferably a value-based method, and make the local kemenization. This procedure possibly reduces the Kendall distance and, thus, provides a sound basis to take decisions.

Finally, with respect to future research work, the final practical decision rules need to be further validated by way of diverse case studies (i.e., with different numbers of components, ranges of the magnitude orders of the component reliabilities, system logic structures, etc.). In this sense, the present work paves the way to a new research pathway on IMs, which builds on the findings of this work (e.g., the relevance of making the local kemenization) to propose further improvements, such as:

- The Footrule aggregation method, which has been investigated by a few authors ([32], [38], [39]), deserves a deeper investigation within the IM context, as its statistical interpretation can support the development of techniques that allow estimating the uncertainties in the final ranking. In this respect, a combination of Bootstrap method and Ensemble-based Sensitivity Analysis (EBSA) has been proposed in [29] to identify the variables that most affect the uncertainty on an output. Similarly, a Bootstrap method has been adopted in [13] for IM RA and also to quantify the uncertainty on each alternative position. However, both works ([13] and [29]) do not consider the aggregation ranking and the concept of distance between rankings. For this, future research work is in order to further capture the statistical interpretation of the outcomes of RA algorithms and, also to give definitive rules to DMs to identify the most important components. This can also constitute the basis of decision algorithms that guide the DM in selecting the portfolio of actions to achieve cost effective improvements of the system RAM attributes.

- In this work, we have disregarded the uncertainty that can affect the component failure probabilities and, thus, the IM values. Indeed, in [13], [41], [42] it has been shown that disregarding these epistemic uncertainties can lead to biased ranking. Future research work will investigate how to aggregate uncertain IMs. For this, we will build on [43][46]: in [43] and [45], Uncertainty IMs (UIMs) have been propounded to quantify the effect of epistemic uncertainties in IMs, which however do not allow tackling the issue of IM ranking in the presence of the epistemic uncertainty. For this, Aven and Nøkland have proposed a ranking method based on the couple (IM, UIM) [46], whereas Toppila and Salo [43] propose a computational method based on a novel branch and bound algorithm to treat multilinear functions of interval-valued probabilities. The objective of the future research work will be the aggregation of these measures to get the final aggregated ranking with the estimation of its robustness.

\section{Conclusion}

RA methods are useful tools to combine the rankings obtained from different IMs and, thus, choose the most impactful components for system reliability. We have presented six different aggregation methods, which have applied to an example of a simplified AFWS in a PWR, composed by fourteen components, with five IMs to aggregate. The aggregation methods have also been compared with the MCDM approach. 
The set of IMs has also been divided into two classes DC and TMC IMs. In all cases, the best methods turn out to be the value-based ones and the Footrule optimal aggregation method (an approximation of the Kemeny method), as they have the smallest Kendall distance from the input IMs. Moreover, the final application of the Local Kemenization procedure seems necessary, when applicable.

Future research works will focus on the formalization of the statistical properties of the Footrule optimal aggregation method and on the characterization of the uncertainty in the final ranking to allow the DM to make more informed decisions.

\section{References}

[1] E. Zio, "An introduction to the basics of reliability and risk analysis", World Scientific Publishing, 2007.

[2] E. Zio, "Computational Methods for Reliability and Risk Analysis", Singapore: World Scientific Publishing, 2009.

[3] W. Kuo, and X. Zhu, "Some Recent Advances on Importance Measures in Reliability," IEEE Transactions on Reliability, vol. 61, no. 2, pp. 344-360, 2012.

[4] Z.W. Birnbaum, On the importance of different components in a multi component system. Multivariate analysis II, New York: Academic Press, 1969.

[5] W. Kuo and X. Zhu, "Relations and generalizations of importance measures in reliability," IEEE Transactions on Reliability, vol. 61, no. 3, pp. 659-674, 2012.

[6] W. Kuo and X. Zhu, "Importance Measures in Reliability, Risk, and Optimization: Principles and Applications", Chichester, UK: John Wiley \& Sons, 2012.

[7] R.W. Youngblood, "Risk significance and safety significance," Reliability Engineering and System Safety, vol. 73, no. 2, pp. 121-136, 2001.

[8] W.E. Vesely, M. Belhadj, and J.T. Rezos, "PRA importance measures for maintenance prioritization applications," Reliability Engineering and System Safety, vol. 43, no. 3, pp. $307-$ 318, 1994.

[9] M.C. Cheok, G.W. Parry, and R.R. Sherry, "Use of importance measures in risk-informed regulatory applications," Reliability Engineering \& System Safety, vol. 60, no. 3, pp. 213226, 1998.

[10] H. Peng, D.W. Coit, and Q. Feng, "Component Reliability Criticality or Importance Measures for Systems With Degrading Components," IEEE Transactions on Reliability, vol. 61 , no. 1, pp. 4-12, 2012

[11] S. Beeson and J.D. Andrews, "Importance Measures for Non-Coherent-System Analysis," IEEE Transactions on Reliability, vol. 42, no. 3, pp. 301-310, 2003

[12] E. Borgonovo and G.E. Apostolakis, "A new importance measure for risk-informed decision making”, Reliability Engineering \& System Safety, vol. 72, no. 2, p. 193, 2001.

[13] Modarres M., "Risk Analysis in Engineering: Techniques, Tools and Trends", Taylor \& Francis Group, 2006.

[14] Cheok M.C., Parry G.W., Sherry R.R., "Use of importance measures in risk informed applications", Reliability Engineering and System Safety, vol. 60, pp. 213-226, 1998.

[15] de Condorcet N., "Essai sur l'application de l'analyse à la probabilité des décisions rendues à la probabilité des voix", Paris, De l'imprimerie royale, 1785. 
[16] Borda J.C. "Mémoire sur les Elections au scrutin", Histoire de l'Académie Royal des Sciences, 1784.

[17] Rousseau J.J., "The social contract”, Harmondsworth, Penguin, 1962.

[18] Boland, P.J. Majority Systems and the Condorcet Jury Problem. Statistician, 30(3), pp. 181-189, 1989.

[19] Pesarin F. and Lago A., "Nonparametric combination of dependent rankings with application to the quality assessment of industrial products", Metron, LVIII, pp. 1-2, 2000.

[20] Shima K., Todoriki M. and Suzuki A., "SVM-based feature selection of latent semantic features," Pattern Recogn. Lett., vol. 25, no. 9, pp. 1051-1057, 2004.

[21] Ekenel H. K. and Sankur B., "Feature selection in the independent component subspace for face recognition," Pattern Recogn. Lett., vol. 25, no. 12, pp. 1377-1388, 2004.

[22] Guyon I. and Elisseeff A., "An introduction to variable and feature selection," J. Mach. Learn. Res., vol. 3, pp. 1157-1182, 2003.

[23] Pihur V., Datta Su. and Datta So., "Weighted rank aggregation of cluster validation measures: a Monte Carlo cross-entropy approach", Bioinformatics, vol. 23 (13), pp. 16071615, 2007.

[24] DeConde R. et al., "Combining results of microarray experiments: a rank aggregation approach", Start Appl Genet Mol Biol, vol. 5, article 15, 2006.

[25] Pihur V., Datta Su. and Datta So., "Finding cancer genes through meta-analysis of microarray experiments: rank aggregation via the cross entropy algorithm", Genomics, vol. 92, pp. 400-403, 2008.

[26] Brin S. and Page L., "The anatomy of a large-scale hypertextual Web search engine", Computer Networks, vol. 30(1-7), pp. 107-117, 1998.

[27] Fagin R., "Combining Fuzzy information from multiple systems", JCSS, vol. 58(1), pp. 83-99, 1999.

[28] Quinn J., Fobes R., "Declaration of Election-Method Reform Advocates", April 2012.

[29] Di Maio, F., Bandini, A. Zio, E., Martorell, S., Carlos Alberola, S. "Bootstrapped Ensemble-based Sensitivity analysis of a TRACE thermal-hydraulic model based on a limited number of PWR large Break LOCA simulations", under review, Reliability Engineering and System Safety.

[30] Ravi Ravindran A., Donald P. and Warsing Jr., "Supply Chain Engineering: Models and Applications", CRC Press, 2012.

[31] Deb, K., Agrawal, S., Pratap, A., Meyarivan, T., "A fast elitist non-dominated soring genetic algorithm for multi-objective optimization: NSGA-II", IEEE Transactions on Evolutionary Computation 6 (2), pp. 182-197, 2002.

[32] Dwork C., Kumar R., Naor M. and Sivakumar D., "Rank aggregation methods for the web", Proceedings of the Tenth International World Wide Web Conference, ACM, 2001, pp. 613-622.

[33] Munkres, J. "Algorithms for the Assignment and Transportation Problems", Journal of the Society for Industrial and Applied Mathematics, Vol. 5, (1), pp. 32-38, 1957.

[34] Burkard, R.E. Dell'Amico, M. Martello S., "Assignment Problems" (Revised reprint), SIAM, Philadelphia (PA), 2012.

[35] Diaconis P. and Graham R., "Spearman's footrule as a measure of disarray", J. of the Royal Statistical Society, Series B, vol. 39(2), pp. 262-268, 1977. 
[36] Papoulis, A. And Pillai S.U. 2002. "Probability, Random Variables and Stochastic Processes", 4th edition, International Edition, Mc-Graw-Hill.

[37] Kemeny J., "Mathematics without Numbers", Daedalus, vol. 88, pp. 571-591, 1959.

[38] Young H. P., "Condorcet's theory of Voting”, Amer. Political Sci. Review, vol. 82, pp. 1231-1244, 1988.

[39] Truchon M., "An extension of the Condorcet criterion", Journal of Economic Literature, 1998.

[40] Diaconis P., "Group Representation in Probability and Statistics", IMS Lectures Series 11, IMS, 1988.

[41] Baraldi, P., Compare, M., Zio, E. "Component ranking by birnbaum importance in presence of epistemic uncertainty in failure event probabilities", IEEE Transactions on Reliability, 62 (1), pp. 37-48, 2013.

[42] Baraldi, P., Zio, E., Compare, M. "A method for ranking components importance in presence of epistemic uncertainties", Journal of Loss Prevention in the Process Industries, 22 (5), pp. 582-592, 2009.

[43] Toppila A., Salo, A. "A computational framework for prioritization of events in fault tree analysis under interval-valued probabilities", IEEE Transactions on Reliability, Vol. 62, No. 3, 2013, pp. 583-595, 2013.

[44] Borgonovo E. "Measuring Uncertainty Importance: Investigation and comparison of alternative approaches", Risk Analysis, vol. 26, no. 5, pp. 1349-1361, 2006.

[45] Borgonovo E., Apostolakis, G. E., Tarantola S., Saltelli, A. "Comparison of global sensitivity analysis techniques and importance measures in PSA", Reliability Engineering and System Safety, vol. 79, no. 2, pp. 175-186, 2003.

[46] Aven, T. Nokland, T. E. "On the use of uncertainty importance measures in reliability and risk analysis", Reliability Engineering and System Safety, vol. 95, no. 2, pp. 127-133, 2010. 